\title{
A reference gene catalogue of chicken gut antibiotic resistomes
}

\section{Jintao Yang}

South China Agricultural University

\section{Cuihong Tong}

South China Agricultural University

\section{Danyu Xiao}

South China Agricultural University

\section{Longfei Xie}

South China Agricultural University

\section{Ruonan Zhao}

South China Agricultural University

Zhipeng Huo

South China Agricultural University

\section{Ziyun Tang}

South China Agricultural University

Jie Hao

South China Agricultural University

\section{Zhenling Zeng}

South China Agricultural University

Wenguang Xiong ( $\nabla$ xiongwg@scau.edu.cn)

South China Agricultural University https://orcid.org/0000-0002-8965-2915

\section{Research}

Keywords: Antibiotic resistance genes, Metagenome, Chicken gut, Host-tracking, Mobile genetic elements

Posted Date: July 7th, 2021

DOI: https://doi.org/10.21203/rs.3.rs-665224/v1

License: (9) This work is licensed under a Creative Commons Attribution 4.0 International License. Read Full License 


\section{A reference gene catalogue of chicken gut antibiotic resistomes}

Jintao Yang ${ }^{1,2,3,4}$, Cuihong Tong ${ }^{1,2,3}$, Danyu Xiao ${ }^{1,2,3}$, Longfei Xie ${ }^{1,2,3}$, Ruonan Zhao ${ }^{1,2,4}$, Zhipeng Huo ${ }^{1,2}$, Ziyun Tang ${ }^{1,2,4}$, Jie Hao ${ }^{1,2,4}$, Zhenling Zeng ${ }^{1,2,3,4 *}$, Wenguang Xiong ${ }^{1,2,3,4^{*}}$

${ }^{1}$ Guangdong Provincial Key Laboratory of Veterinary Pharmaceutics Development and Safety Evaluation, College of Veterinary Medicine, South China Agricultural University, Guangzhou 510642, China

${ }^{2}$ Guangdong Laboratory for Lingnan Modern Agriculture, Guangzhou 510642, China

${ }^{3}$ National Laboratory of Safety Evaluation (Environmental Assessment) of Veterinary Drugs, College of Veterinary Medicine, South China Agricultural University, Guangzhou 510642, China

${ }^{4}$ National Risk Assessment Laboratory for Antimicrobial Resistance of Animal Original Bacteria, College of Veterinary Medicine, South China Agricultural University, Guangzhou 510642, China

E-mail addresses:

Jintao Yang,708948663@qq.com; Cuihong Tong, 1197522867@qq.com; Danyu Xiao, 865907461@qq.com; Longfei Xie, 2911922454@qq.com; Ruonan Zhao, 1804581643@qq.com; Zhipeng Huo, 979817515@qq.com; Ziyun Tang, 1598387644@qq.com; Jie Hao, 1284634903@qq.com; Zhenling Zeng, zlzeng@scau.edu.cn; Wenguang Xiong, xiongwg@scau.edu.cn

*Corresponding author: zlzeng@scau.edu.cn; xiongwg@scau.edu.cn 


\begin{abstract}
Background: The chicken gut microbiota, as a reservoir of antibiotic resistance genes (ARGs), poses a high risk to humans and animals worldwide. Yet a comprehensive exploration of the chicken gut antibiotic resistomes remains incomplete.

Results: In this study, we established the largest chicken gut resistance gene catalogue to date through metagenomic analysis of 629 chicken gut samples. We found significantly higher abundance of ARGs in the Chinese chicken gut than that in the Europe. tet $X, m c r$, and bla $a_{\mathrm{NDM}}$, the genes resistant to antibiotics of last resort for human and animal health, were frequently detected in the Chinese chicken gut. The abundance of ARGs was linearly correlated with that of mobile genetic elements (MGEs). The host-tracking analysis identified Escherichia, Enterococcus, Staphylococcus, Klebsiella, and Lactobacillus as the major ARG hosts. Especially, Lactobacillus, an intestinal probiotic, carried multiple drug resistance genes, and was proportional to ISLhe63, highlighting its potential risk in agricultural production processes.
\end{abstract}

Conclusions: We first established a reference gene catalogue of chicken gut antibiotic resistomes. Our study help to improve the knowledge and understanding of chicken antibiotic resistomes for knowledge-based sustainable chicken meat production.

Keywords: Antibiotic resistance genes, Metagenome, Chicken gut, Host-tracking, Mobile genetic elements

\title{
Introduction
}

Antibiotic resistance has been recognized as a significant global threat to public health and food safety by the World Health Organization[1]. Currently, hundreds of thousands of people die each year from infections caused by antibiotic-resistant bacteria. Irrational use of antibiotics is thought to be the main driver for the development of antibiotic resistance[2]. Antibiotic resistance genes (ARGs) are widely distributed in surface water, sewage treatment plant effluent, soil, and animal feces[3]. In livestock farming environments, ARGs and drug-resistant bacteria can be 
transmitted to humans through the food chain, water, or air. Besides, the horizontal gene transfer (HGT) process makes it possible for bacteria from various ecological niches to share genes, thereby playing a vital role in the dissemination and evolution of ARGs. Undoubtedly, the gut atmosphere offers a strong communication site. A significant number of ARGs have been identified in the poultry gut[4].

As the world's population grows, the demand for animal protein including poultry meat, as a high-quality protein, is increasing day by day. Broiler chickens are considered one of the world's main meat suppliers. Global poultry meat consumption increased from $11 \mathrm{~kg}$ per capita in 2000 to $14,4 \mathrm{~kg}$ per capita in 2011 and is projected to reach $17,2 \mathrm{~kg}$ per capita in 2030 [5]. The production of poultry meat will reach 134.5 million tons in 2023, according to a report submitted by the Organization for Economic Cooperation and Development and the Food and Agriculture Organization[6]. The modernization of livestock farming is inseparable from the use of antibiotics, and it is estimated that the global consumption of antimicrobials will increase by $67 \%$ between 2010 and 2030[7]. The continued use of antibiotics would alter the microbial structure of the animal gut and cause an increase in abundance of antibiotic resistance genes in the animal gut. This anthropogenic activity promotes the emergence and spread of antibiotic resistance genes including tet $X, m c r$, and bla $a_{\mathrm{NDM}}$, the genes resistant to antibiotics of last resort for human and animal health.

Tigecycline is one of the last resorts for the treatment of complex infections caused by multi-resistant Gram-negative and Gram-positive bacteria. In 2019, two variants of plasmid-encoded tet $X$, named tet $X 3$ and tet $X 4$, were identified in Enterobacter and Acinetobacter isolated from animal and human sources. Plasmids carrying tet $X 3$ and tet $X 4$ are already widely distributed in human pathogens and are transferable and stable[8]. Polymyxin is an important lipopeptide antibiotic that serves as the last line of defense against multidrug-resistant gram-negative bacterial infections[9]. In November 2015, the first plasmid-mediated polymyxin resistance gene, $m c r-1$, was discovered in China[10]. $m c r-1$ and its variants have been identified in more than 50 countries to date. The $m c r-1$ gene is carried by multiple plasmids, including IncX4, IncHI2, and IncI2, which strengthens the risk of further $m c r-1$ 
transmission worldwide. The bla $a_{\mathrm{NDM}}$ gene encodes a carbapenemase that hydrolyzes almost all $\beta$-lactam antibiotics. Carbapenem-resistant Enterobacteriaceae that produce the bla $a_{\mathrm{NDM}}$ gene are found worldwide and have become a major public health threat.

Due to the diversity and abundance of microorganisms in the gut microecology, the human and animal gut is an important site for the increase and spread of ARGs, with a large number of ARGs settling and spreading to the gut flora through host cells. Currently, studies of gut resistance groups have been conducted in a variety of animals, such as humans, pigs, and chickens[11], which have deepened the understanding of the distribution, evolution, and transmission of ARGs in the gut flora. However, in general, there is still no standard process for metagenomic analysis.

In this study, we explored the comprehensive profile of ARGs in chicken gut by using large-scale metagenomic datasets. We established the largest chicken gut resistance gene catalogue to date through metagenomic analysis of 629 chicken gut samples. We analyzed the diversity, abundance of ARGs and ARG hosts from multiple perspectives. Besides, we reconstructed 4062 high-quality metagenome-assembled genomes (MAGs) using a binning approach, most of which belong to species lacking culture representation. Combining these individual efforts and establishing a unified non-redundant dataset of the chicken gut genome is essential to advance future microbiome studies. We think these data will lay the foundation for future studies on the structure of chicken gut microbiota.

\section{Methods and materials}

\section{Metagenomic sample collection}

The 36 gut samples of chicken were collected from our previous study[12]. There were 60 chicken gut samples from the current laboratory study. A total of 533 chicken gut samples from 11 countries were randomly selected from NCBI, including China $(n=344)$, Europe $(n=178)$, including Netherlands $(n=20)$, Germany $(n=19)$, France $(n=20)$, Spain $(n=20)$, Belgium $(n=20)$, Italy $(n=20)$, Poland $(n=20)$, Denmark $(n=20)$ and Bulgaria $(n=19)$ and USA $(n=11)$. Furthermore, all the above 
metagenomic datasets that were sequenced using the Illumina HiSeq platform with PE150 (paired-end sequencing $150 \times 2$ ) were downloaded to avoid the fluctuations caused by different sequencing strategies. Additional sample descriptions can be found in Supporting Information Fig. 1 and Table S1.

\section{Quality control and metagenome assembly}

First, raw reads with average quality scores $<20(\mathrm{Q} 20)$ or length $<20$ bp (L20) were removed using Sickle[13] (https://github.com/najoshi/sickle). After that, the metagenomic sequencing data of each sample were de novo assembled with default k-mer size using the CLC Genomics Workbench (Version 10.0.1, CLC Bio, Aarhus, Denmark), and the assembled contigs longer than $500 \mathrm{bp}$ were reserved. We finally obtained 35672711 contigs from 629 metagenomic samples. The detailed information is summarized in Table S2.

\section{Identification of ARG-like ORFs and the mobile genetic elements (MGEs)}

The open reading frames (ORFs) of the contigs were predicted using Prodigal v2.6.3 with a metamodel [14]. The ARG-like ORFs were identified against the resfinder database [15] using BLASTN with an E-value of $\leq 1 \times 10^{-5}$, and the results that identity $\geq 80 \%$ and over $70 \%$ query coverage were identified as ARG-like ORFs[16]. The coverage of ARG-like ORFs was calculated by mapping metagenomic reads to the contigs with a minimum length coverage of $95 \%$ at $95 \%$ similarity and contigs minimum length $\geq 500 \mathrm{bp}$ using the CLC Genomics Workbench[16]. The number of cells in each metagenome sample was calculated by ARGs-OAP v2.0[17] (Table S1 for complete sample cell count information). To compare the coverage of each different sample, the ARG-like ORFs coverage was normalized by the number of cells in each sample (copies/cell). The calculation formula was as follows:

$$
\text { Coverage }=\sum_{1}^{\mathrm{n}} \frac{N \times 150 / L}{C}
$$

where $\mathrm{N}$ is the number of reads mapped to ARG-like ORFs, $\mathrm{L}$ is the sequence length of the target ARG-like ORFs, $\mathrm{n}$ is the number of ARG-like ORFs, and 150 is the length of Illumina sequencing reads $[12,16]$, and the $\mathrm{C}$ means the cell number of per sample. 
Likewise, the MGEs database including plasmid, IS, and Integron was identified against the plasmidfinder[18], ISfinder[19], and Integron[20] databases, respectively. The final abundance calculation method was the same as the above equation remains the same.

\section{Phylogenetic analyses}

All phylogenetic trees were inferred using MEGA X (Version 10.1.8) with 1000 bootstraps[21]. To construct phylogenetic relationships of important ARGs, the corresponding sequences of $\operatorname{tet} X(\mathrm{n}=136), \operatorname{mcr}(\mathrm{n}=22)$, and bla $a_{\mathrm{NDM}}(\mathrm{n}=13)$ were analyzed, separately. The protein of ORF was aligned using clustalw. The phylogenetic tree was plotted with iTol (Version 5.7)[22].

\section{Identification of bacterial host of ARG-carrying contigs}

The predicted protein sequences of ORFs from the contigs that carried ARG-like ORFs were annotated using BLASTP against non-redundant protein NCBI databases at $E$-value $\leq 1 \times 10^{-5}[23]$. The results were annotated using ETE Toolkit (version 2.3) for the assignment of taxonomic phylum and genus [24]. A contig was assigned to the taxon if it obtained more than $50 \%$ of the vote [25].

\section{Genome reconstruction of gut microbes and genome annotation of MAGs}

With the functional module of metaWRAP (v1.2.1), a pipeline that contains several modules for the study of metagenomic bins[26], genome reconstruction of gut microbes using metagenomic sequencing data is carried out. Briefly, the metagenome-assembled genomes (MAGs) were constructed using contigs by the binning algorithm "MetaBAT2"[27] in the metaWRAP software. The default of the minimum length of contigs used for constructing bins with MetaBAT2 was $1500 \mathrm{bp}$.

CheckM v1.1.2 with options lineage_wf, - $x$ fa was used to assess the completeness and contamination of all MAGs[28]. After filtering for completeness $\geq$ $80 \%$ and contamination $\leq 10 \%[29]$, we were left with 4062 high-quality bins from the 629 samples. These high-quality bins were annotated to the genus using the Genome Taxonomy Database Toolkit (GTDB-Tk)[30].

\section{Statistical analysis}

The heatmap, correlation heatmap, and variance analysis were generated using 
the pheatmap, ggcorrplo, and limma packages in the $\mathrm{R}$ package (v4.0.2, https://www.r-project.org/), respectively. The alpha diversity of the gut microbiota, including the number of species and the Shannon index, was calculated by spaa and vagen in the R package (v4.0.2). MetaPhlan2 (v2.7.5)[31] was used to identify species and to determine their relative abundances across samples. The network was explored and visualized using the interactive platform of Gephi (v0.9.2). The drawing of simple graphs was done by GraphPad Prism 8. Statistical analyses were performed using SPSS software (PASW Statistics 22.0). The Pearson correlation coefficient was used to show the correlation between the abundance of ARGs and the abundance of MGEs. A two-tailed Wilcoxon test (two-by-two comparison) was used to compare Chinese and European chickens. A $P$-value $<0.05$ was considered a statistically significant difference. The Procrustes transformation was performed using two Bray-Curtis distance plots (PCoA) as input based on microbial community matrices and ARGs at the subtype level. R (v4.0.2), Python (v3.7.3) programs were used to handle the text processing, information extraction, and data statistics for this study.

\section{Results}

\section{Broad-spectrum profile of ARG abundance}

In total, 387ARG subtypes belonging to $14 \mathrm{ARG}$ types were detected in at least one of the 629 chicken gut samples, with total abundances ranging from 0.031 to 3.42 $\times 10$ copies/cell. The resistance genes to tetracycline (34.24\%), aminoglycoside (19.37\%), macrolide (19.37\%), phenicol (10.94\%), sulphonamide (5.46\%), beta-lactam $(5.09 \%)$, oxazolidinone $(1.88 \%)$, quinolone $(1.70 \%)$ were more abundant and prevalent in these samples.

Depending on the geographical location of the samples, we could divide them into two groups, China and Europe (Due to the small number of samples in the USA, there were no group discussions). Overall, the average abundance of ARG types in the chicken gut was 3.62 copies/cell. The tetracycline (1.24 copies/cell), aminoglycoside (0.70 copies/cell), and macrolide (0.69 copies/cell) resistance genes were the predominant ARG types. (Fig. 2b). The abundance of ARG types in China was 
significantly different from that in Europe $(P<0.0001)$. The average abundance of ARGs was about three times higher in China than that in Europe (Fig. 2c). Human activities (different strategies for antibiotic use in different geographic areas) may be the main driver of the different distribution of ARGs.

The similarity of ARG subtypes compositions was calculated using principal component analysis (PCA). Chicken gut samples were well clustered in Europe, whereas in China they were more dispersed and well surrounded by European samples, suggesting that the Chinese region has a more diverse ARG composition than that in Europe (Fig. 2a). Each sample carried a different number of species of ARGs, so we further compared the diversity of ARGs between China and Europe. Interestingly, the results showed the presence of more diverse resistance gene types in Europe (Fig. 2d and Fig. 2e). This may be related to the fact that the samples collected in Europe were spread over nine countries.

Of the 387 ARG subtypes detected in the chicken gut, the top five abundant ARG subtypes were considered representative ARGs, tetW, ermB, tetA, tetL, and sul2. Interestingly, we found that more than half of these five dominant ARG subtypes were tetracycline resistance genes, of which tet $W$ accounted for $18.25 \%$ of the overall.

The tet $X, m c r$, and bla $a_{\mathrm{NDM}}$ were all detected to varying degrees in 629 chicken gut samples. The tet $X$ was detected more frequently in European chicken gut samples (93 of $178,3.78 \times 10^{-4}-1.18 \times 10^{-1}$ copies/cell) than that in Chinese (17 of 440, $6.93 \times 10^{-3}-3.67 \times 10^{-1}$ copies/cell). Besides, the $\mathrm{mcr}$ was detected more frequently in Chinese chicken gut samples ( 70 of $440,1.61 \times 10^{-3}-1.63 \times 10^{-1}$ copies/cell) and to a lesser extent in European chicken gut samples (13 of 178, $1.23 \times 10^{-3}-5.99 \times 10^{-2}$ copies/cell). Also, we found that bla $a_{\mathrm{NDM}}$ was only detected in Chinese chicken gut samples $\left(11\right.$ of $440,2.79 \times 10^{-3}-2.51 \times 10^{-1}$ copies/cell). Furthermore, we obtained further distributions by constructing a phylogenetic tree analysis of these genes. Concerning tet $X$ genes, tet $X 10$ and tet $X 2$ were more prevalent in the chicken gut and most were present on plasmids. Most of tet $X$ belonged to the Bacteroides and Butyricimonas. IS4351, IS1187, IS612, IS614, and ISBfl1 may have an important role in the transfer of tet $X$. Most of $m c r-1$ were present on plasmids that were attributed to 
Escherichia coli, Escherichia albertii, and Klebsiella pneumonia. As well, ISApl1 was the predominant transposon for $m c r-1$ transfer. Most of bla $a_{\mathrm{NDM}}$ were also present on plasmids carried by a variety of pathogenic bacteria such as E. coli, Salmonella enterica, and Proteus mirabilis. ISABa125, IS5D, and IS26 were potential for their transfer vectors (Fig. 3).

\section{Bacterial community structure}

Overall, the Firmicutes (59.13\%), Proteobacteria (25.38\%), Bacteroidetes (7.05\%), Actinobacteria (5.36\%) were the predominant phyla in the chicken gut samples. While there was no variation between the Chinese and European samples in the dominant bacterial phylum (Fig. 4b). Lactobacillus and Escherichia had the highest abundance percentages of $42.47 \%$ and $18.43 \%$, respectively. It was hypothesized that the changes in the abundance of Lactobacillus and Escherichia in the chicken gut were the main reasons for the changes in the percentages of their corresponding phyla. Interestingly, we find that the European samples cluster together, while the Chinese samples wrap around the European samples (Fig. 4a), which is highly similar to the previous ARG subtypes compositions. Lactobacillus and Escherichia were the two genera with the most significant differences between the Chinese and European groups, respectively (Fig. 4c). The correlation analysis revealed that the abundance of Lactobacillus and Escherichia were negatively correlated $(\rho=-0.503, P<0.01$, Spearman's correlation coefficient, two-tailed test).

Previous studies have shown that bacterial community structure determines the distribution of resistance genes without considering HGT, and a significant correlation between bacterial composition and ARG profiles could observe [32, 33]. Visualized by the Procrustes analyses, ARGs and bacterial composition in the chicken gut showed highly significant goodness of fit by ANOVA $\left(\mathrm{M}^{2}=0.7888, P<0.001\right)$ (Fig. S1).

\section{The host of ARGs in chicken gut samples}

After filtering the contigs with lengths less than $500 \mathrm{bp}$, we obtained a total of 35672711 contigs from 629 samples, of which 24318 contigs were identified as ARG-carrying contigs (ACCs) (Table S2). Only 10809 (44.5\%) of these contigs could 
be annotated to the genus level (244 genera), suggesting that we have limited knowledge of the hosts carrying ARGs.

Overall, at the phylum level, Firmicutes (42.6\%), Proteobacteria $(28.7 \%)$, and Bacteroidetes (4.8\%) were the major ARG carriers (Table S6). At the genus level, Escherichia (6.3\%), Enterococcus (5.3\%), Staphylococcus (5.1\%), Klebsiella (3.2\%), Lactobacillus (2.6\%), Ligilactobacillus (2.1\%), Limosilactobacillus (1.8\%), Corynebacterium (1.7\%), Bacteroides (1.6\%) and Acinetobacter (1.1\%) were the main ARGs hosts (Fig. 5). Besides, we found that the dominant genus Acinetobacter carries sulphonamide resistance genes. Wang et al. also identified a novel strain capable of degrading sulfamethoxazole in 2018, identified as Acinetobacter sp[34]. Escherichia was the most common carrier of ARGs. Escherichia had a high percentage of $94.00 \%$ for colistin resistance genes, in addition to the highest percentage for aminoglycosides and trimethoprim resistance genes with $28.89 \%$ and $33.07 \%$, respectively. All glycopeptide resistance genes were found in Enterococcus, while Enterococcus had the highest percentage of resistance genes for macrolides, oxazolidinones, and chloramphenicol with $15.25 \%, 94.44 \%$, and $16.35 \%$, respectively. Klebsiella pneumoniae was also found to have the highest proportion of resistance genes in phosphomycin, quinolone, and rifampicin with $52.36 \%, 67.84 \%$, and $93.18 \%$, respectively. Bacillus spp. had the highest percentage of sulfonamide resistance genes (53.78\%), and Bacillus spp. had the highest percentage of $\beta$-lactam and nitroimidazole resistance genes (23.04\% and $73.33 \%$, respectively). Lactobacillus, as a probiotic, was also the genus that accounted for more of the macrolide and tetracycline, accounting for $8.19 \%$ and $12.27 \%$, respectively (Table S3). The predominant genera of Bacillus, Aspergillus, E. coli, Klebsiella, and Staphylococcus that carry ARGs are mostly considered as conditional pathogens, and their ability to colonize the chicken gut may increase the exposure and transmission of ARGs.

\section{MGEs}

To better evaluate the potential transferability of ARGs in chicken gut samples, we tracked the ARGs associated with MGEs. Overall, the average abundance of MGEs in the chicken gut was 8.01 copies/cell. We identified a total of 10971 contigs 
carrying integrator sequences, 19830 contigs carrying insertion sequences, and 8589 contigs carrying plasmid fragments, which have great HGT potential. Meanwhile, we further screened the ARGs that co-occurred with MGEs (ARGs have at least one MGE next to them on the contig) that have been identified for mobilized ARGs[35], and we found 6409 (29.91\%) contigs with coexisting ARGs and MGEs out of 21431 ACCs.

The phylum with the highest percentage of these 6409 contigs with coexisting ARGs and MGEs was Proteobacteria (62.16\%), while at the level of the genus, the highest percentage was Escherichia (12.65\%), followed by Klebsiella(6.54\%). Also in terms of the types of drug resistance included, the highest percentage was aminoglycosides $(26.56 \%)$, followed by phenicol, $\beta$-lactam, and sulfonamide with $16.54 \%, 13.90 \%$, and $13.48 \%$, respectively. We further explored the correlation between intI1 and ARGs. intII was found to be correlated with aph(6)-Id, bla OXA-10, bla ${ }_{\mathrm{TEM}-1 \mathrm{~B}}, \mathrm{mph} A$, floR, qnrS1, sul1, sul2, sul3, and tetA, which are drug resistance gene subtypes significant positive correlations (Table S4).

We found a linear correlation between the abundance of MGEs and the abundance of ARGs $\left(y=3.15+1.35 \mathrm{x}, \mathrm{R}^{2}=0.3\right)$ (Fig. 6a), and also the correlation heat map produced by ggcorrplot package showed a correlation between ARGs and MGEs ( $\mathrm{r}=0.56, P<0.05)($ Fig. $6 \mathrm{c})$, indicating that the presence of MGEs plays a transfer of ARGs an important role. We found the highest abundance of ISLhe63 among the IS and ISLHe63 showed a significant correlation with Lactobacillus $(\rho=0.621, P<0.05)$, which is consistent with previous studies[36].

Using ARGs structure as the response variable, the top 10 genus in terms of abundance and 3 types of MGEs(Integron, IS, Plasmid) as explanatory variables for RDA, after forward model selection, provided 12 explanatory variables, all of which were significantly positively correlated except for Bacteroides(Fig. S2a). VPA was used to determine the co-occurrence of MGEs and genus on ARGs structure. The results showed that the most important influences on the structure of ARGs were MGEs, with individual explanatory rates of $12.1 \%$ and $6.6 \%$, respectively(Fig. S2b).

\section{MAGs from the chicken gut}


On each of the 629 samples, we carried out a single-sample metagenomic assembly. A total of 25598 MAGs were created from binning. With a total of 4062 high-quality genomes with completeness $\geq 80 \%$ and contamination $\leq 10 \%$, we were left. Of these 1823 had completeness $>90 \%$ and contamination $<5 \%, 938$ were $>$ $95 \%$ complete with $<5 \%$ contamination, and 163 MAGs were $>97 \%$ complete with $0 \%$ contamination. The distribution of these MAGs between the 629 samples can be found in Table S5.

Table S5 contains the GTDB-TK classification of each high-quality MAGs, and Fig. S3 shows the phylogenetic tree of MAGs, which helps to further correct the occurrence of classification errors. Overall, the most dominant clade was Firmicutes $(\mathrm{n}=2551)$, followed by Bacteroidota $(\mathrm{n}=803)$, Actinobacteriote $(\mathrm{n}=345)$, Proteobacteria $(\mathrm{n}=176)$, Campylobacterota $(\mathrm{n}=71)$, Desulfobacterota $(\mathrm{n}=57)$, and so on We also identified four archaea, one UBA71 sp006954425, two Methanobrevibacter_A woesei and one Methanocorpusculum.

Previous research analyzing the possible hosts of ARGs in metagenomic data usually used the method of correlation network analysis[37], but this solution has the downside that only a small number of ARG hosts can be revealed and further confirmation is required. A recent study has explored potential hosts by ARGs-like-ORFs in assembled contigs[38]. However, only partial hosts of ARGs could be revealed and a large number of ARGs were not precisely assigned to genera. Therefore, we explored ARG potential hosts from multiple perspectives by ARGs-like-ORFs in assembled contigs and by using binning to obtain MAGs. We further screened the high-quality MAGs and obtained 655 MAGs carrying ARGs. As shown in Fig. 7, Enterococcus carries various ARGs such as glycopeptides (vanC1XY, vanC4XY and vanHAX), oxazolidinones (optrA), and tetracyclines (tet $X$ and tetX10). Klebsiella pneumoniae carried fosfomycin (fosA6) and E. coli carried $\beta$-lactams (bla $a_{\text {СтХ-м-65 and bla }}$ СтХ-м-55). It was also observed that Lactobacillus carried mainly macrolides (lnuA and vatE, etc.)

Due to the limitations of the taxonomically annotated reference sequences, some MAGs failed to classify to the genus level. This limitation is common in the 
taxonomic identification of genomes generated from metagenomic data[29]. Despite this, this study still revealed that 156 bacteria genera were the hosts of 140 ARG subtypes. Therefore, this study greatly expands the knowledge of the diversity of ARBs in the chicken gut.

\section{Discussion}

Our study analyzed antibiotic resistance based on metagenomic tools within 629 chicken gut samples, demonstrating the correlation between ARGs and MGEs and revealing ARG hosts from multiple perspectives. The microbiome in the chicken gut microbiota constitutes a large reservoir of ARGs. Tetracyclines, aminoglycosides, and macrolides were the most commonly observed ARG forms in this study, whereas certain resistance genes are often present in particular habitats, such as urban waste[39], human intestine[40]. However, the major ARGs in this study differed from the most frequently occurring bacitracin in drinking water[38], and also from the soil[32], sludge[33]. This suggests that ARGs are not randomly distributed in different environments.

We found that the total level of acquired antimicrobials correlates with the overall livestock antimicrobial use. The average ARG abundance in the gut of chickens in China was three times higher than that in Europe. According to a survey report by the World Organization for Animal Health (OIE) in February 2019, Asia, the Far East, and Oceania had the highest average use of antimicrobials in animals in the world, amounting to $296.75 \mathrm{mg} / \mathrm{kg}$, which is 3.74 times higher than in Europe. This value is close to the average abundance ratio of ARGs between China and Europe above. The total ban of all antibiotic growth promoters in the EU member states was in 2006 while it in China was in 2020. Different strategies of human antibiotic use in agricultural practices may be responsible for this phenomenon.

ARGs were frequently detected in the chicken gut, such as tet $X, m c r$, and bla $a_{\mathrm{NDM}}$, which may be due to the selection posed by prolonged exposure of gut microbes to antibiotics. Although tigecycline was not applied to livestock animal farming, tetX was still found in the gut environment of chickens in this study, presumably due to the 
selection pressure of tetracyclines. The latest appearance from animals and humans of plasmid-mediated high levels of tigecycline resistance genes tet $X 3$ and tet $X 4$ has triggered the much broader public alarm[8], rendering it important for robust surveillance of tet $X$ genes to be improved. In November 2015, the plasmid-mediated polymyxin resistance gene $m c r-1$ was first identified, subsequently, multiple Enterobacteriaceae members with different host origins were found carrying the resistance gene in more than 50 countries per continent, suggesting that $m c r-1$ was spread globally[41]. And it has been demonstrated that the transposon part Tn6330 (ISAPl1-mcr-1-orf-ISAPl1) can be removed from the plasmid to form an ISAPl1-mcr-1-orf intermediate loop which can be inserted into other ISAPl1-carrying plasmids[42, 43]. Meanwhile, the gut flora can act as a suitable mixing vessel to facilitate the spread of $m c r$ through horizontal gene transfer[43, 44]. bla $a_{\mathrm{NDM}}$ genes showed a highly variable region upstream of ISAba125, IS26, and IS5D. The highly conserved bla $a_{\mathrm{NDM}-5}$ genetic surrounding structures [DISAba125-bla $\mathrm{NDM}_{\mathrm{N}-\mathrm{-}} \mathrm{ble}$ MBL-trpT-dsbD-IS26] were found in German and Swiss E. coli of both the F-type and IncX3 plasmids[45-47]. But for the potential transfer between IS5D and bla $a_{\mathrm{NDM}}$ has not been reported. The tet $X, m c r$, and $b l a_{\text {NDM }}$ genes in the chicken gut, especially in China, were frequently detected in the present study. This poses a significant risk to the use of drugs in agricultural practices and deserves our attention. Furthermore, the evidence is growing that genes with antibiotic resistance can be transferred from animals to humans[48]. During homeotic development, tracking of tet $X$, $m c r$, and bla $_{\mathrm{NDM}}$ in the gut should be stressed.

The ARGs present in the MGEs undergoes constant horizontal shifts through human induction and environmental changes[49], which is following Darwinian evolutionary theory. In this study, MGEs were also present in $29.91 \%$ of ACCs. We found that the relative abundance of MGEs was linearly correlated with that of ARGs. As the key reason for their survival and spread, horizontal transmission of MGEs to a wide variety of bacteria (including pathogens and human commensals) has been established[50]. A large amount of ARGs is stored in the gut of chickens, which may undergo horizontal gene transfer via MGEs and is most likely to be transmitted to 
humans by food chain or spread in the environment, such as rivers and soil, via the fecal route[51], indicating the potential risk for human health. To improve monitoring of ARGs and MGEs in the gut tract of chickens and proper handling of livestock manure, this needs to be a concern.

We constructed 4062 high-quality MAGs from 629 chicken gut samples, greatly extending the enrichment of previous chicken gut MAGs[29, 52]. Reconstruction of microbial genomes using metagenomic assembly has been applied to humans[53, 54], cattle[55], pigs[56], the bank voles [57], soil[58], and ocean[59], but less in the chicken gut. We found several new strains of Lactobacillus, including L.gallinarum, L.crispatus, and L.johnsonii. We also identified several new strains of Bifidobacterium, including B. gallinarum, B. globosum, and B. animalis. These strains are considered to be potential chicken gut probiotics[60]. It has been shown that probiotics can reduce pathogen transfer in the intestinal mucosa by enhancing intestinal barrier integrity and maintaining immune tolerance. At the level of genus, the most significant difference was Escherichia in China and Lactobacillus in Europe. It has been shown that probiotics (especially Lactobacillus) have preventive and protective effects against Salmonella enterica and Escherichia coil O78:K80 infections in chickens[61, 62]. Although a variety of Lactobacillus is generally considered non-pathogenic for use in a variety of human and animal foods and products, they have a tremendous role in maintaining gut health, such as the bioactive Lactobacillus casei competitively reducing avian gut bacterial pathogens[63]. However, it has also been shown that multiple ARGs are isolated from Lactobacillus strains and some of them may even transfer their intrinsic ARGs to other lactic acid bacteria or pathogens through horizontal gene transfer[64-67]. In our study, Lactobacillus was also found to carry a variety of ARGs, especially macrolides and tetracyclines. This suggests that Lactobacillus can potentially act as a reservoir of ARGs and may play an active role in the transfer of ARGs to humans through the food chain. We identified 4 archaea in the 4062 high-quality MAGs. It has been shown that Methanobrevibacter and Methanocorpusculum were positively correlated with all of the detected ARGs[68]. Although we did not identify any ARGs in the 4 
archaeal strains, it still provides a new idea for a potential link between archaea and ARGs.

\section{Conclusion}

We established the largest chicken gut resistance gene catalogue to date through metagenomic analysis of 629 chicken gut samples and revealed the diversity and abundance of ARGs in the chicken gut. The results explained that the differences in the abundance levels of ARGs (e.g., tet $X, m c r$, and bla $a_{\mathrm{NDM}}$ ) between different regions in China and Europe were completely positively correlated with anthropogenic factors. The widespread presence of MGEs further drives the spread of ARGs through horizontal transmission. Lactobacillus, as gut probiotics, also carried multiple drug resistance genes (especially macrolides and tetracyclines), emphasizing their potential risk. Besides, the number of chicken gut microbial genomes in public databases was enriched by reconstructing a large number of MAGs, but a whole-genome sequencing approach after isolation and culture is still needed to validate the description. Our study helps to improve the knowledge and understanding of chicken gut flora and promote knowledge-based sustainable chicken production. 


\section{Abbreviations}

ARGs: Antibiotic resistance genes; MGEs: Mobile gene elements;

ACCs: ARG-carrying contigs; HGT: Horizontal gene transfer;

MAGs: Metagenome-assembled genomes; ORFs: open reading frames;

E. coli: Escherichia coli

\section{Declarations}

Ethics approval and consent to participate

Not applicable.

\section{Consent for publication}

Not applicable.

\section{Data availability}

The dataset supporting the conclusions of this article is included within its supplementary file.

\section{Competing interests}

The author declares that have no competing interests.

\section{Funding}

This work was supported by the National Natural Science Foundation of China (no. $31702290)$.

\section{Authors' contributions}

J.Y. prepared figures and wrote the manuscript. J.Y., D.X., L.X., R.Z., and C.T. analyzed the data Z.T., J.H. and Z.H. collected samples. W.X. and Z.Z. conceived the study. All authors edited the manuscript and approved the final draft.

\section{Acknowledgments}

The authors are grateful to the anonymous reviewers for their insightful suggestions and comments. 


\section{References}

1. Nathan C, Cars O: Antibiotic resistance--problems, progress, and prospects. New England Journal of Medicine 2014, 371(19):1761-1763.

2. Bengtsson-Palme J, Kristiansson E, Larsson DJ: Environmental factors influencing the development and spread of antibiotic resistance. Fems Microbiology Reviews 2018.

3. Qiao M, Ying GG, Singer AC, Zhu YG: Review of antibiotic resistance in China and its environment. Environ Int 2018, 110:160-172.

4. Wang Y, Na L, Liu F, Liu WJ, Gao GF: More diversified antibiotic resistance genes in chickens and workers of the live poultry markets. Environment International 2021, 153(1791-1795):106534.

5. Gao R, Hu Y, Li Z, Sun J, Wang Q, Lin J, Ye H, Liu F, Srinivas S, Li D et al: Dissemination and Mechanism for the MCR-1 Colistin Resistance. PLOS Pathog 2016, 12(11):e1005957.

6. Muaz K, Riaz M, Akhtar S, Park S, Ismail A: Antibiotic Residues in Chicken Meat: Global Prevalence, Threats, and Decontamination Strategies: A Review. J Food Prot 2018, 81(4):619-627.

7. Boeckel TPV, Brower C, Gilbert M, Grenfell BT, Laxminarayan R: From the Cover: Global trends in antimicrobial use in food animals. Proceedings of the National Academy of Sciences 2015, 112(18)

8. He T, Wang R, Liu D, Walsh TR, Zhang R, Lv Y, Ke Y, Ji Q, Wei R, Liu Z et al. Emergence of plasmid-mediated high-level tigecycline resistance genes in animals and humans. Nat Microbio/ 2019, 4(9):1450-1456.

9. Nang SC, Li J, Velkov T: The rise and spread of mcr plasmid-mediated polymyxin resistance. Crit Rev Microbio/ 2019, 45(2):131-161.

10. Wang R, Dorp LV, Shaw LP, Bradley P, Balloux F: The global distribution and spread of the mobilized colistin resistance gene mcr-1. Nature Communications 2018, 9(1):1179.

11. Wang Y, Hu Y, Liu F, Cao J, Lv N, Zhu B, Zhang G, Gao GF: Integrated metagenomic and metatranscriptomic profiling reveals differentially expressed resistomes in human, chicken, and pig gut microbiomes. Environ Int 2020, 138:105649.

12. Xiong $W$, Wang $Y$, Sun $Y$, Ma L, Zeng $Q$, Jiang $X, L i ~ A$, Zeng $Z$, Zhang $T$ : Antibiotic-mediated changes in the fecal microbiome of broiler chickens define the incidence of antibiotic resistance genes. Microbiome 2018, 6(1):34.

13. Joshi N, Fass J: Sickle: a sliding-window, adaptive, quality-based trimming tool for FastQ files. 2011.

14. Hyatt D, Chen GL, Locascio PF, Land ML, Larimer FW, Hauser LJ: Prodigal: prokaryotic gene recognition and translation initiation site identification. BMC Bioinformatics 2010, 11:119.

15. Zankari E, Hasman H, Cosentino S, Vestergaard M, Rasmussen S, Lund O, Aarestrup FM, Larsen MV: Identification of acquired antimicrobial resistance genes. J Antimicrob Chemother 2012, 67(11):2640-2644.

16. Liping, Ma, Yu, Xia, Bing, Li, Ying, Yang, Li-Guan, James: Metagenomic Assembly Reveals Hosts of Antibiotic Resistance Genes and the Shared Resistome in Pig, Chicken, and Human Feces. Environmental science \& technology 2016.

17. Yin X, Jiang XT, Chai B, Li L, Yang Y, Cole JR, Tiedje JM, Zhang T: ARGs-OAP v2.0 with an 
expanded SARG database and Hidden Markov Models for enhancement characterization and quantification of antibiotic resistance genes in environmental metagenomes. Bioinformatics 2018, 34(13):2263-2270.

18. Lund O, Villa L, Aarestrup FM, Hasman H: PlasmidFinder and pMLST: in silico detection and typing of plasmids. antimicrobial agents \& chemotherapy.

19. Siguier P, Perochon J, Lestrade L, Mahillon J, Chandler M: ISfinder: the reference centre for bacterial insertion sequences. Nucleic Acids Res 2006, 34(Database issue):D32-36.

20. Yan B, Labbate M, Koenig JE, Stokes HW: Integrons: mobilizable platforms that promote genetic diversity in bacteria. Trends in Microbiology 2007, 15(7):301-309.

21. Kumar S, Stecher G, Li M, Knyaz C, Tamura K: MEGA X: Molecular Evolutionary Genetics Analysis across Computing Platforms. Mol Biol Evo/2018, 35(6):1547-1549.

22. Letunic I, Bork P: Interactive tree of life (iTOL) v3: an online tool for the display and annotation of phylogenetic and other trees. Nucleic Acids Res 2016, 44(W1):W242-245.

23. Mao $Y$, Xia $Y$, Wang $Z$, Zhang $T$ : Reconstructing a Thauera genome from a hydrogenotrophic-denitrifying consortium using metagenomic sequence data. Applied Microbiology \& Biotechnology 2014, 98(15):6885-6895.

24. Huson DH, Mitra S, Ruscheweyh HJ, Weber N, Schuster SC: Integrative Analysis of Environmental Sequences Using MEGAN4. Genome Research 2011, 21(9):1552-1560.

25. Ishii S, Suzuki S, Norden-Krichmar TM, Tenney A, Chain PS, Scholz MB, Nealson KH, Bretschger O: A novel metatranscriptomic approach to identify gene expression dynamics during extracellular electron transfer. Nat Commun 2013, 4:1601.

26. Gherman, Uritskiy, Jocelyne, DiRuggiero, James, Taylor: MetaWRAP-a flexible pipeline for genome-resolved metagenomic data analysis. Microbiome 2018.

27. Kang DD, Li F, Kirton E, Thomas A, Wang Z: MetaBAT 2: An adaptive binning algorithm for robust and efficient genome reconstruction from metagenome assemblies. Peer 2019, 7(7):e7359.

28. Parks DH, Imelfort M, Skennerton CT, Hugenholtz P, Tyson GW: CheckM: assessing the quality of microbial genomes recovered from isolates, single cells, and metagenomes. Genome Res 2015, 25(7):1043-1055.

29. Glendinning L, Stewart RD, Pallen MJ, Watson KA, Watson M: Assembly of hundreds of novel bacterial genomes from the chicken caecum. Genome Bio/2020, 21(1):34.

30. Chaumeil PA, Mussig AJ, Hugenholtz P, Parks D: GTDB-Tk: a toolkit to classify genomes with the Genome Taxonomy Database. Bioinformatics 2019, 36(6).

31. Tin D, Truong, Eric A, Franzosa, Timothy L, Tickle, Matthias, Scholz, George, Weingart: MetaPhIAn2 for enhanced metagenomic taxonomic profiling. Nature methods 2015.

32. Forsberg KJ, Patel S, Gibson MK, Lauber CL, Knight R, Fierer N, Dantas G: Bacterial phylogeny structures soil resistomes across habitats. Nature 2014, 509(7502):612-616.

33. Cda B, Yu XB, Mz C, Tang LA, Wen LA, Qian CA, Jn A: Metagenomic insights into the profile of antibiotic resistomes in a large drinking water reservoir. Environment International, 136.

34. Wang S, Wang J: Biodegradation and metabolic pathway of sulfamethoxazole by a novel strain Acinetobacter sp. App/ Microbiol Biotechno/2018, 102(1):425-432. 
35. Ju F, Beck K, Yin X, Maccagnan A, McArdell CS, Singer HP, Johnson DR, Zhang T, Burgmann $\mathrm{H}$ : Wastewater treatment plant resistomes are shaped by bacterial composition, genetic exchange, and upregulated expression in the effluent microbiomes. ISME J 2019, 13(2):346-360.

36. Callanan M, Kaleta P, O'Callaghan J, O'Sullivan O, Ross RP: Genome Sequence of Lactobacillus helveticus, an Organism Distinguished by Selective Gene Loss and Insertion Sequence Element Expansion. Journal of Bacteriology 2008, 190(2):727-735.

37. Zeng J, Pan $Y$, Yang J, Hou M, Zeng Z, Xiong W: Metagenomic insights into the distribution of antibiotic resistome between the gut-associated environments and the pristine environments. Environment international 2019, 126:346-354.

38. Ma L, Li B, Jiang XT, Wang $Y L, X i a ~ Y, L i ~ A D$, Zhang $T$ : Catalogue of antibiotic resistome and host-tracking in drinking water deciphered by a large scale survey. Microbiome 2017, 5(1):154.

39. Hendriksen RS, Munk P, Njage P, van Bunnik B, McNally L, Lukjancenko O, Roder T, Nieuwenhuijse D, Pedersen SK, Kjeldgaard J et al: Global monitoring of antimicrobial resistance based on metagenomics analyses of urban sewage. Nat Commun 2019, 10(1):1124.

40. Forsberg KJ, Reyes A, Wang B, Selleck EM, Sommer M, Dantas G: The Shared Antibiotic Resistome of Soil Bacteria and Human Pathogens. Science 2012, 337(6098):1107-1111.

41. Giamarellou H: Epidemiology of infections caused by polymyxin-resistant pathogens Int J Antimicrob Agents 2016, 48(6):614-621.

42. Li R, Xie M, LV J, Chan WC, Chen S: Complete genetic analysis of plasmids carrying mcr-1 and other resistance genes in an Escherichia coli isolate of animal origin. Journal of Antimicrobial Chemotherapy 2016:696.

43. Han H, Liu W, Cui X, Cheng X, Jiang X: Co-Existence of mcr-1 and bla NDM-5 in an Escherichia coli Strain Isolated from the Pharmaceutical Industry, WWTP. Infect Drug Resist 2020, 13:851-854.

44. Li XP, Sun RY, Song JQ, Fang LX, Zhang RM, Lian XL, Liao XP, Liu YH, Lin J, Sun J: Within-host heterogeneity and flexibility of mcr-1 transmission in chicken gut. Int J Antimicrob Agents 2020, 55(1):105806.

45. María P, Sola C, Adriana O, Verónica B, Sara M, Guillermo RC, Jesus M, González-Barberá E, Concepción G, Belén A: Emergence of NDM-producing Klebsiella pneumoniae and Escherichia coli in Spain: phylogeny, resistome, virulence and plasmids encoding blaNDM-like genes as determined by WGS. Journal of Antimicrobial Chemotherapy 2019(12):12.

46. Weber RE, Pietsch M, Frühauf A, Pfeifer Y, Fuchs S: IS26-Mediated Transfer of blaNDM-1 as the Main Route of Resistance Transmission During a Polyclonal, Multispecies Outbreak in a German Hospital. Frontiers in Microbiology 2019, 10:2817.

47. Chakraborty T, Sadek M, Yao Y, Imirzalioglu C, Stephan R, Poirel L, Nordmann P: Cross-border emergence of Escherichia coli producing the carbapenemase NDM-5 in Switzerland and Germany. J Clin Microbio/ 2020.

48. Nuria, Vieco-Saiz, Yanath, Belguesmia, Ruth, Raspoet, Eric, Auclair, Frédérique, Gancel: Benefits and Inputs From Lactic Acid Bacteria and Their Bacteriocins as Alternatives 
to Antibiotic Growth Promoters During Food-Animal Production. Frontiers in microbiology 2019.

49. Marta P, Magdalena P: Insight into the mobilome of Aeromonas strains. Frontiers in Microbiology 2015, 6(494).

50. Checcucci A, Trevisi P, Luise D, Modesto M, Mattarelli P: Exploring the Animal Waste Resistome: The Spread of Antimicrobial Resistance Genes Through the Use of Livestock Manure. Frontiers in Microbiology 2020, 11.

51. Zhou SY, Zhu D, Giles M, Yang XR, Daniell T, Neilson R, Zhu YG: Phyllosphere of staple crops under pig manure fertilization, a reservoir of antibiotic resistance genes. Environ Pollut 2019, 252(Pt A):227-235.

52. Sergeant MJ, Constantinidou C, Cogan TA, Bedford MR, Pallen MJ: Extensive Microbial and Functional Diversity within the Chicken Cecal Microbiome. Plos One 2014, 9(3):e91941.

53. Parks DH, Rinke C, Chuvochina M, Chaumeil PA, Woodcroft BJ, Evans PN, Hugenholtz P, Tyson GW: Recovery of nearly 8,000 metagenome-assembled genomes substantially expands the tree of life. Nature Microbiology 2018.

54. ndre Ax, Almeida, Alex, L., Mitchell, Miguel, Boland, Samuel, C., Forster: A new genomic blueprint of the human gut microbiota. Nature 2019, 568(7753):499-504.

55. Wilkinson $T$, Korir D, Ogugo M, Stewart RD, Robert C: 1200 high-quality metagenome-assembled genomes from the rumen of African cattle and their relevance in the context of sub-optimal feeding. Genome biology 2020, 21(1):229.

56. Chen C, Zhou Y, Fu H, Xiong X, Fang S, Jiang H, Wu J, Yang H, Gao J, Huang L: Expanded catalog of microbial genes and metagenome-assembled genomes from the pig gut microbiome. Nat Commun 2021, 12(1):1106.

57. Lavrinienko A, Tukalenko E, Mousseau TA, Thompson LR, Knight R, Mappes T, Watts PC: Two hundred and fifty-four metagenome-assembled bacterial genomes from the bank vole gut microbiota. Sci Data 2020, 7(1):312.

58. Nascimento Lemos L, Manoharan L, William Mendes L, Monteiro Venturini A, Satler Pylro $\checkmark$, Tsai SM: Metagenome assembled-genomes reveal similar functional profiles of CPR/Patescibacteria phyla in soils. Environ Microbiol Rep 2020, 12(6):651-655.

59. Tully BJ, Graham ED, Heidelberg JF: The reconstruction of 2,631 draft metagenome-assembled genomes from the global oceans. Sci Data 2018, 5:170203.

60. Cisek AA, Binek M: Chicken intestinal microbiota function with a special emphasis on the role of probiotic bacteria. Pol J Vet Sci 2014, 17(2):385-394.

61. Carey CM, Kostrzynska M, Ojha S, Thompson S: The effect of probiotics and organic acids on Shiga-toxin 2 gene expression in enterohemorrhagic Escherichia coli O157:H7. J Microbiol Methods 2008, 73(2):125-132.

62. Maragkoudakis PA, Mountzouris KC, Psyrras D, Cremonese S, Fischer J, Cantor MD, Tsakalidou E: Functional properties of novel protective lactic acid bacteria and application in raw chicken meat against Listeria monocytogenes and Salmonella enteritidis. Int J Food Microbio/ 2009, 130(3):219-226.

63. Tabashsum Z, Peng M, Alvarado-Martinez Z, Aditya A, Bhatti J, Romo PB, Young A, Biswas D: Competitive reduction of poultry-borne enteric bacterial pathogens in chicken gut with bioactive Lactobacillus casei. Sci Rep 2020, 10(1):16259. 
64. Guo H, Pan L, Li L, Lu J, Kwok L, Menghe B, Zhang H, Zhang W: Characterization of Antibiotic Resistance Genes from Lactobacillus Isolated from Traditional Dairy Products. J Food Sci 2017, 82(3):724-730.

65. Li Y, Li L, Kromann S, Chen M, Shi L, Meng H: Antibiotic Resistance of Lactobacillus spp. and Streptococcus thermophilus Isolated from Chinese Fermented Milk Products. Foodborne Pathog Dis 2019, 16(3):221-228.

66. Hazirolan G, Gundogdu A, Nigiz S, Altun B, Gur D: Presence of OXA-48 Gene in a Clinical Isolate of Lactobacillus rhamnosus. Foodborne Pathog Dis 2019, 16(12):840-843.

67. Vieira D, Roque R, Moreira JS, Resende D, Nicoli JR, Neumann E, Nunes ÁC: Transfer of antibiotic resistance determinants between lactobacilli isolates from the gastrointestinal tract of chicken. Beneficial Microbes 2012, 3(2):137-144.

68. Fan H, Wu S, Woodley J, Zhuang G, Bai Z, Xu S, Wang X, Zhuang X: Effective removal of antibiotic resistance genes and potential links with archaeal communities during vacuum-type composting and positive-pressure composting. J Environ Sci (China) 2020, 89:277-286. 


\section{Supplementary Information}

Table S1. Sample information.

Table S2. Information on contigs and ORFs.

Table S3. Host (genus level) distribution of ARG types (\%).

Table S4. Correlation of intII with 10 ARGs subtypes.

Table S5. A detailed description of high-quality MAGs.

Table S6. Genus annotation level of ARG-carrying contigs and their classification of ARG-carrying bacteria.

Fig. S1 Procrustes analysis depicts the correlation between ARG content (Bray-Curtis) and bacterial community (Bray-Curtis), $\mathrm{M} 2=0.7888, \mathrm{P}<0.001$.

Fig. S2 (a) RDA plot reveal the relationship between MGEs and major Genus on ARGs. All of the variables in the model were evaluated for collinearity using the variance inflation factor. $* * * P<0.001, * * P<0.01, * P<0.05$ (b) Variation partitioning analysis of the effects of MGEs and major Genus on the ARGs.

Fig. S3 Phylogenetic tree of 4058 (excluding four archaeal strains) MAGs in the chicken gut, labeled according to the taxonomic order defined by GTDB-TK, with different colors in the branch background representing the phylum.

\section{Figure Legends}

Fig. 1 Distribution map of chicken gutl samples and diversity of ARGs, pie chart showing the profile of ARG abundance (top 3 ARG types). Europe(n=178) includes Netherlands(n=20), Germany $(n=19)$, France $(n=20), \operatorname{Spain}(n=20), \operatorname{Belgium}(n=20)$, $\operatorname{Italy}(n=20)$, Poland $(n=20)$, Denmark $(n=20)$ and Bulgaria $(n=19)$.

Fig. 2 ARG composition structure distribution. (a) PCA plots showing the ARG subtypes composition differences among the 618 chicken gut samples. (b) Comparison of ARG types in China and Europe. (c) ARGs abundance comparison. (d) Diversity of ARGs subtypes. (e) ARGs subtype of Shannon's Diversity Index

Fig. 3 ARGs heatmap and phylogenetic tree of tet $X, m c r$, and bla $a_{\mathrm{NDM}}$ detected in the chicken gut.

Fig. 4 Bacterial population structure distribution. (a) PCA plots showing the Bacterial 
flora (genus level) composition differences among the 618 chicken gut samples. (b) Comparison of Bacterial flora in China and Europe. (c) Differentially abundant bacteria. Differences in genus abundance between China and Europe. The y-axis represents the log2-fold change and the $\mathrm{x}$-axis represents the genus name.

Fig. 5 The taxonomy of ACCs (in genus level) and the percentages of ARG types these contigs carried. For example, Escherichia (6.3\%) represents that $6.3 \%$ of ACCs were annotated as Escherichia. b Bar chart shows the percentages of ARG types that were carried by the annotated ACCs.For example, $44.1 \%$ of the ARG-carrying contigs originating from Escherichia carried aminoglycoside resistance genes.

Fig. 6 Correlation between ARGs and MGEs.(a)Overall correlation of ARGs abundance and MGEs abundance in 629 chicken gut samples(b)Correlation of ARGs abundance and MGEs abundance by Chinese and European groups. (c)Heatmap of correlation between MGEs, IS, plasmid and integron.

Fig. 7 The network analysis revealing the co-occurrence patterns between major MAGs(in genus level) and ARG subtypes. The nodes were colored according to the modularity class. Modularity index 0.68 . The size of each node was proportional to the number of connections, that is, the average weighted. 


\section{Figures}

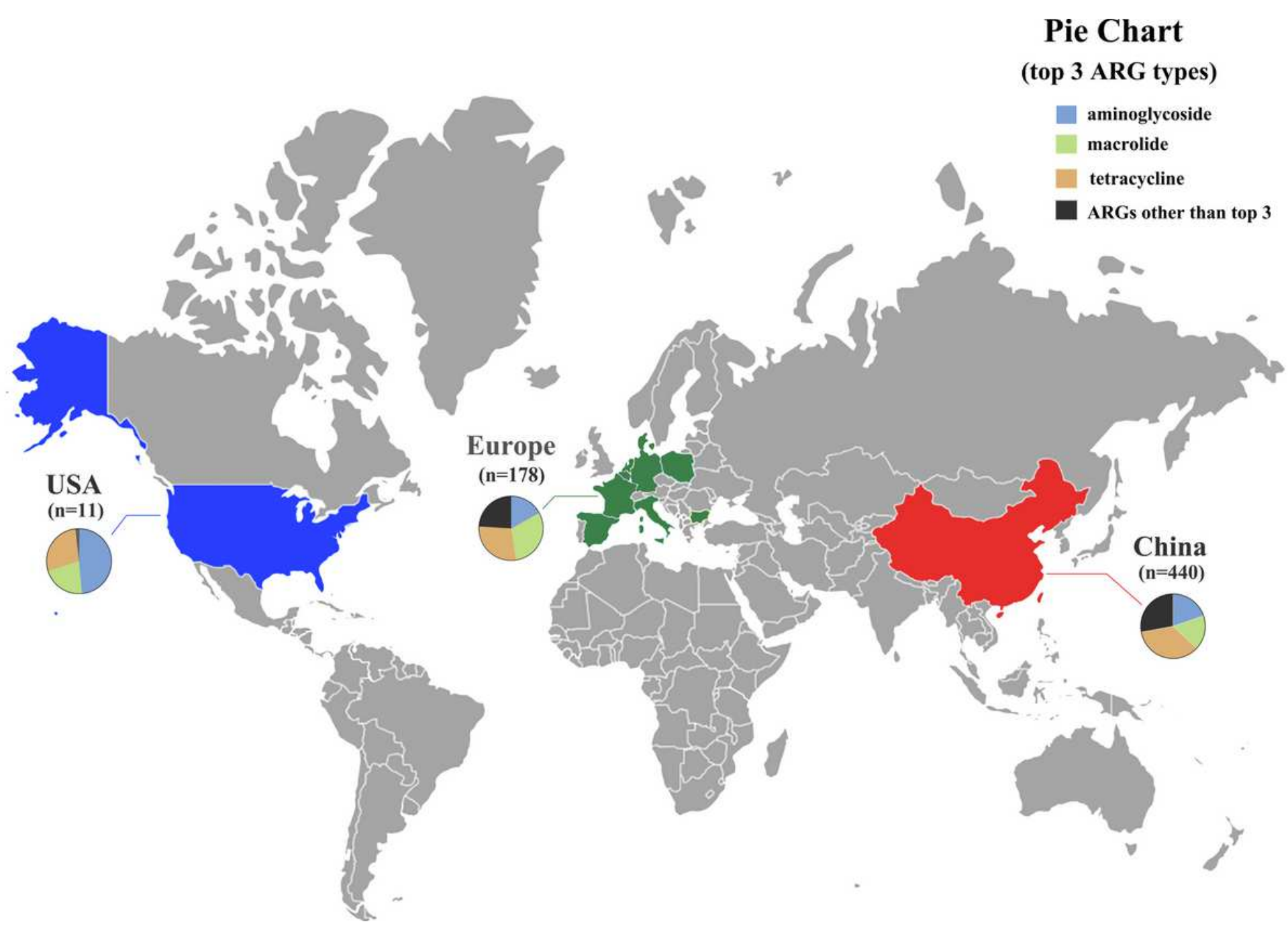

\section{Figure 1}

Distribution map of chicken gutl samples and diversity of ARGs, pie chart showing the profile of ARG abundance (top 3 ARG types). Europe( $n=178)$ includes Netherlands $(n=20)$, Germany $(n=19)$, France $(n=20)$, Spain $(n=20)$, Belgium $(n=20)$, Italy $(n=20)$, Poland $(n=20)$, Denmark $(n=20)$ and Bulgaria $(n=19)$. 


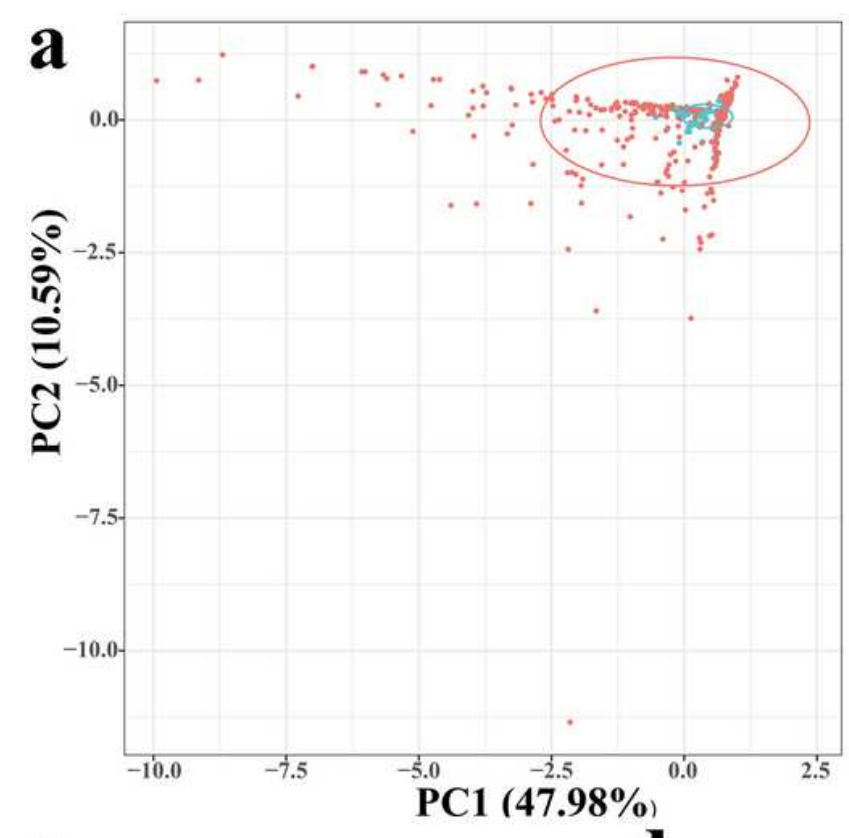

c

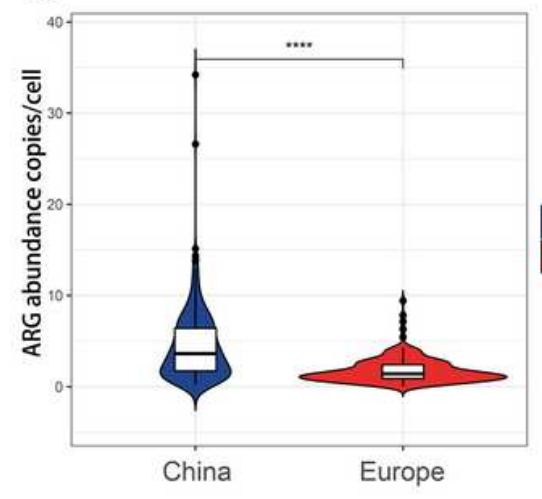

b

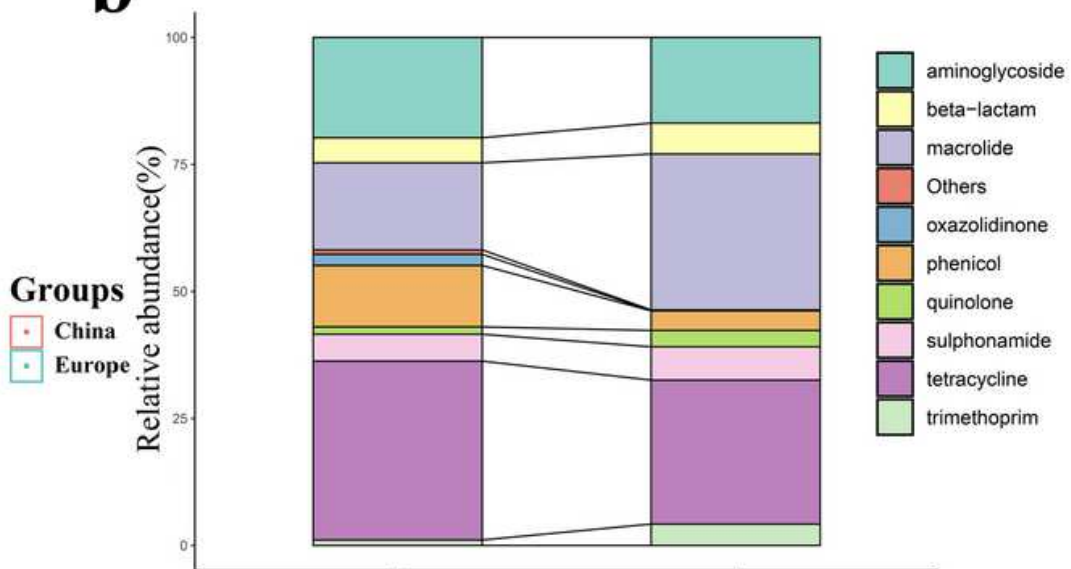

China
Europe

e
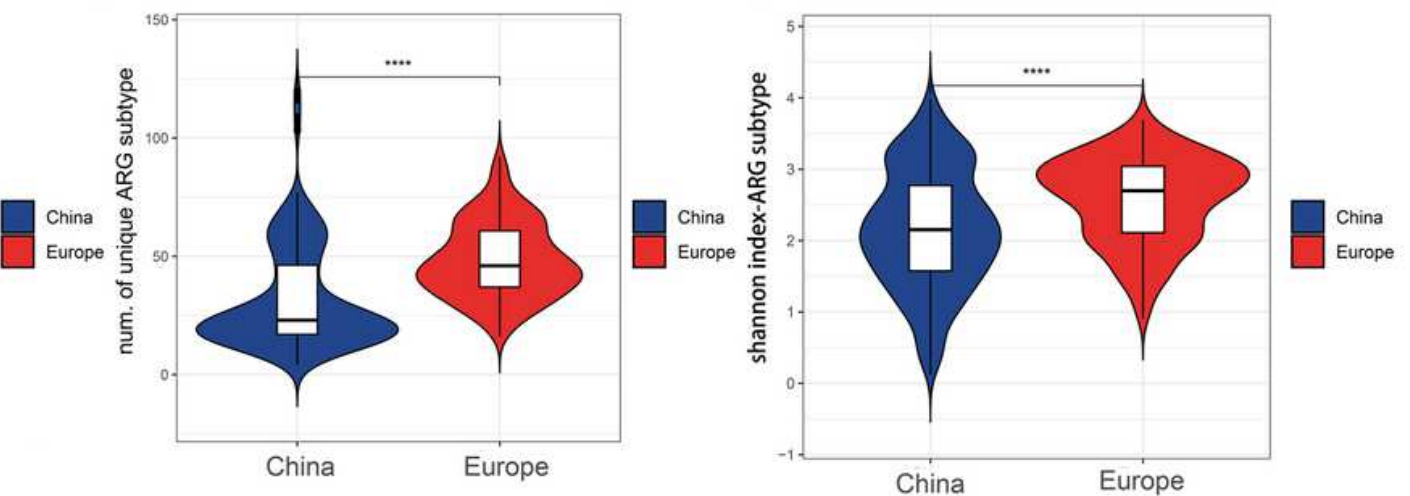

Figure 2

ARG composition structure distribution. (a) PCA plots showing the ARG subtypes composition differences among the 618 chicken gut samples. (b) Comparison of ARG types in China and Europe. (c) ARGs abundance comparison. (d) Diversity of ARGs subtypes. (e) ARGs subtype of Shannon's Diversity Index 


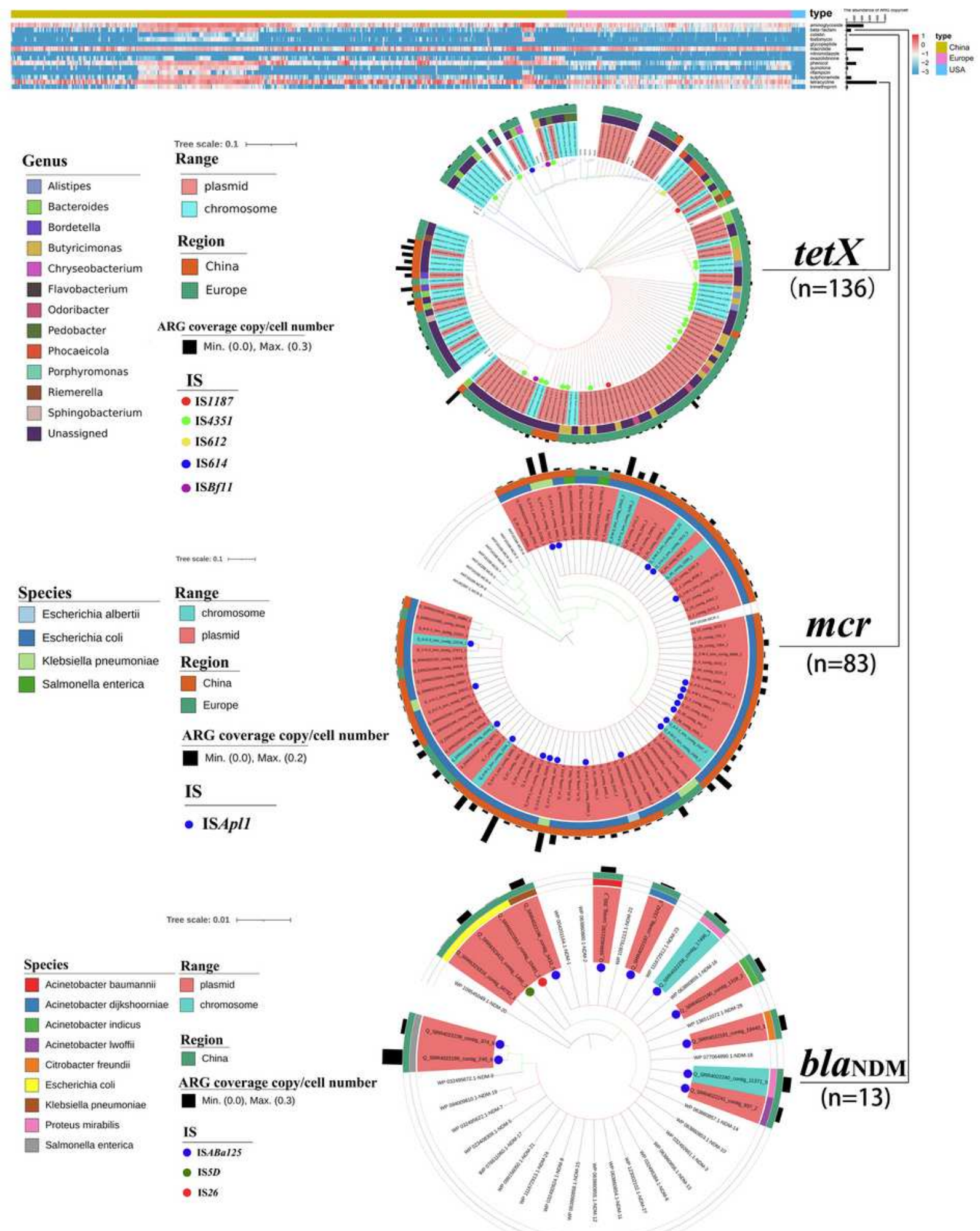

Figure 3

ARGs heatmap and phylogenetic tree of tetX, mcr, and blaNDM detected in the chicken gut. 

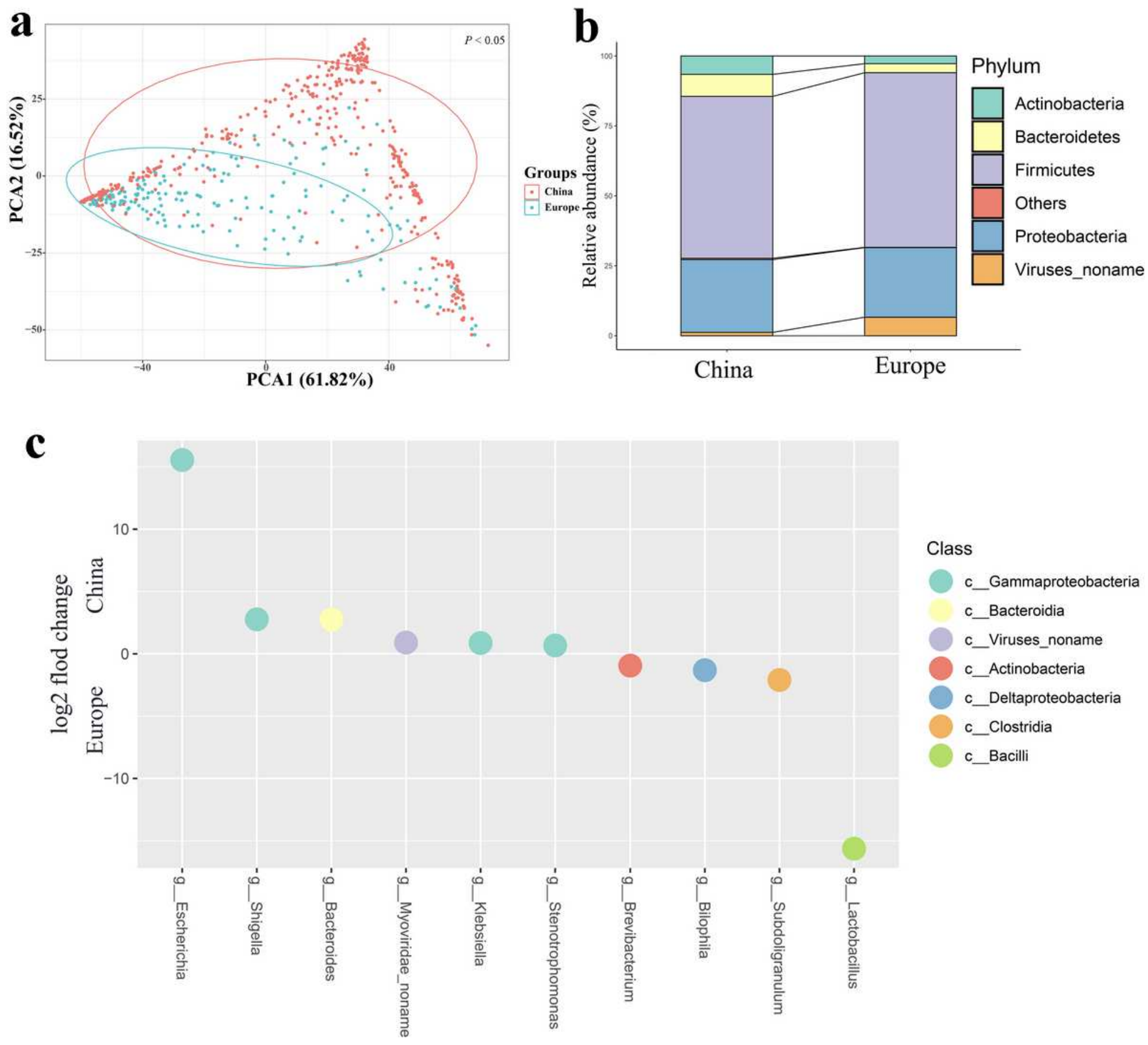

Figure 4

Bacterial population structure distribution. (a) PCA plots showing the Bacterial flora (genus level) composition differences among the 618 chicken gut samples. (b) Comparison of Bacterial flora in China and Europe. (c) Differentially abundant bacteria. Differences in genus abundance between China and Europe. The $y$-axis represents the log2-fold change and the $x$-axis represents the genus name. 


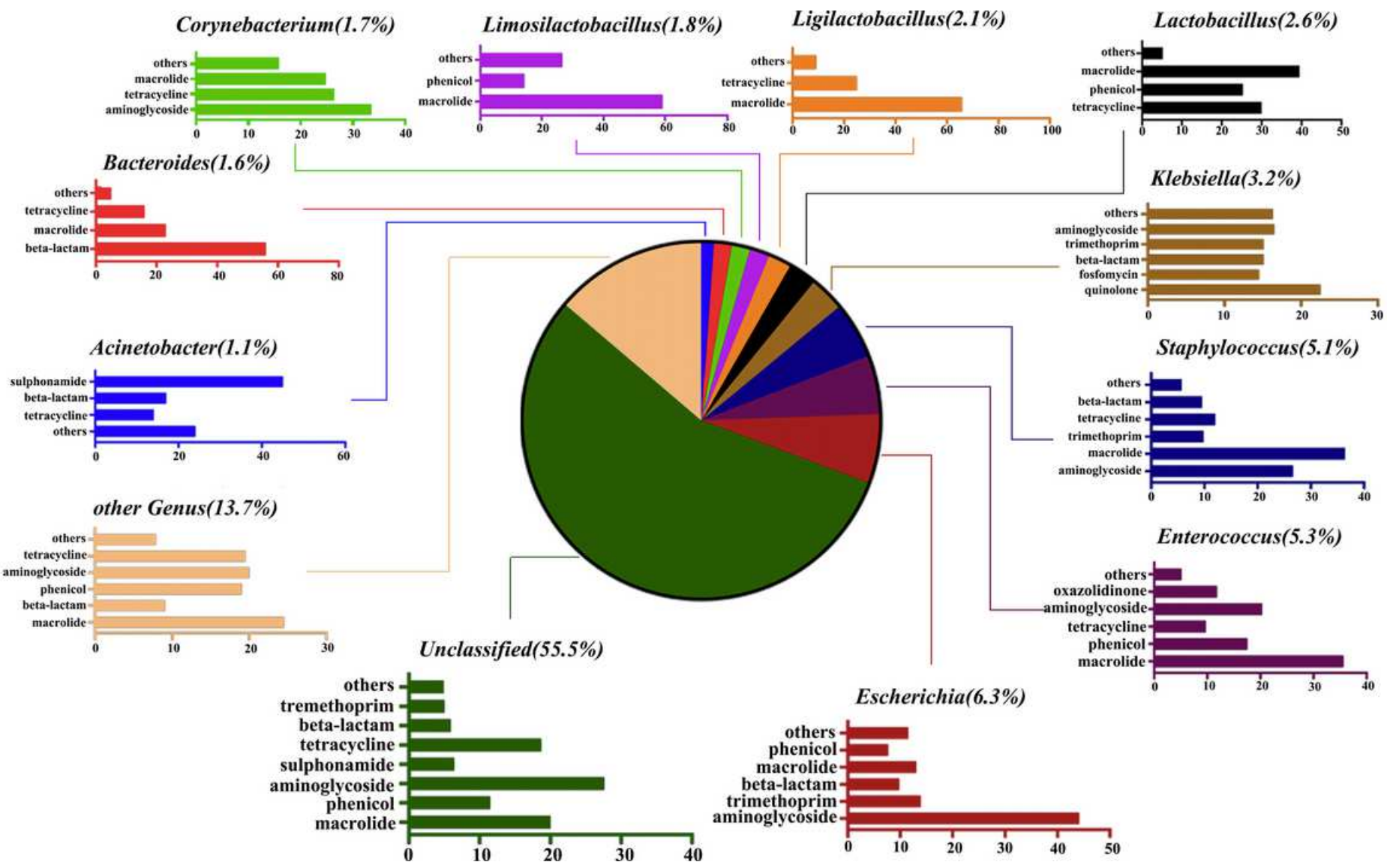

Figure 5

The taxonomy of ACCs (in genus level) and the percentages of ARG types these contigs carried. For example, Escherichia (6.3\%) represents that $6.3 \%$ of ACCs were annotated as Escherichia. b Bar chart shows the percentages of ARG types that were carried by the annotated ACCs.For example, $44.1 \%$ of the ARG-carrying contigs originating from Escherichia carried aminoglycoside resistance genes. 

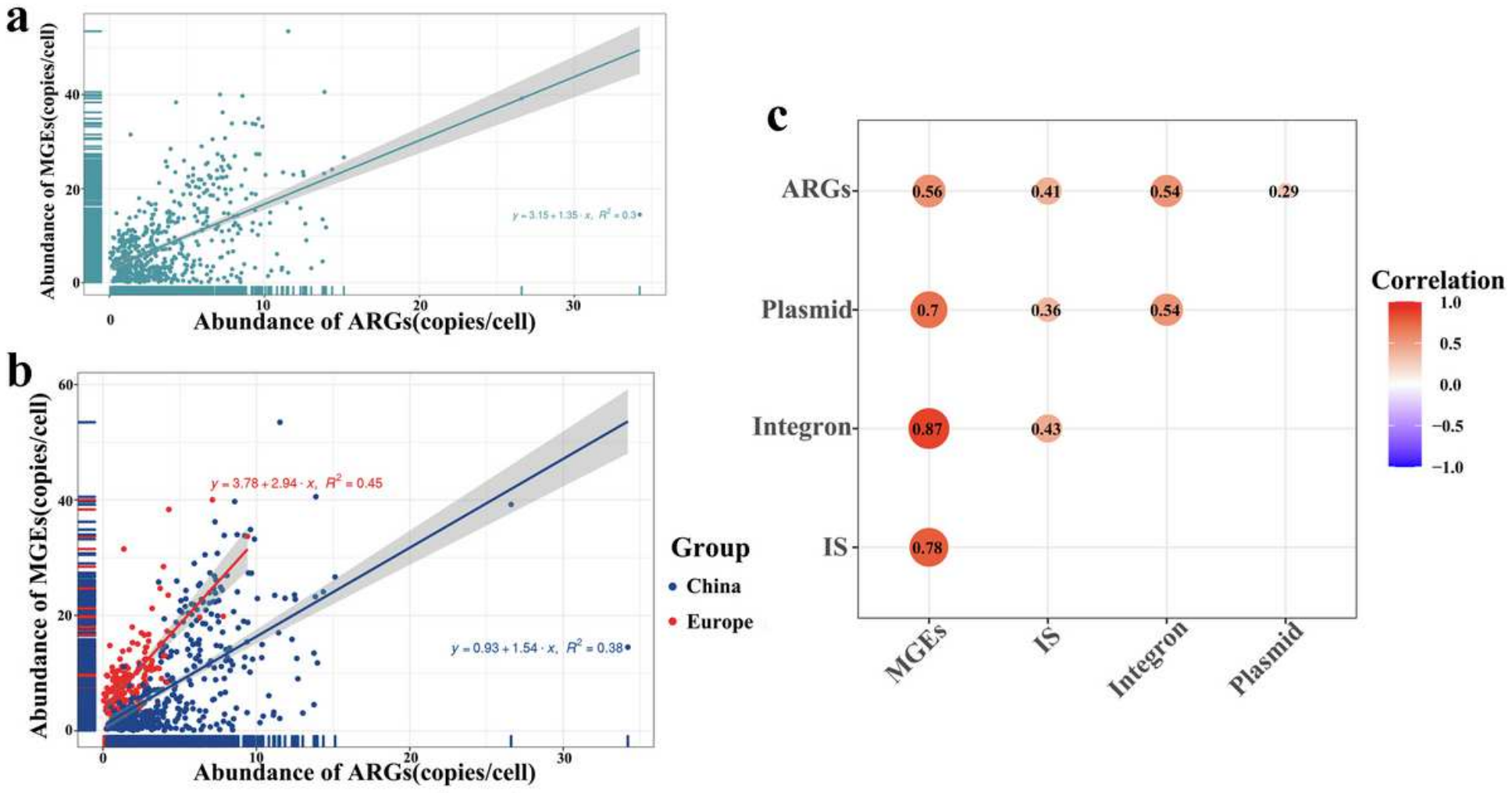

Figure 6

Correlation between ARGs and MGEs.(a)Overall correlation of ARGs abundance and MGEs abundance in 629 chicken gut samples(b)Correlation of ARGs abundance and MGEs abundance by Chinese and European groups. (c)Heatmap of correlation between MGEs, IS, plasmid and integron. 


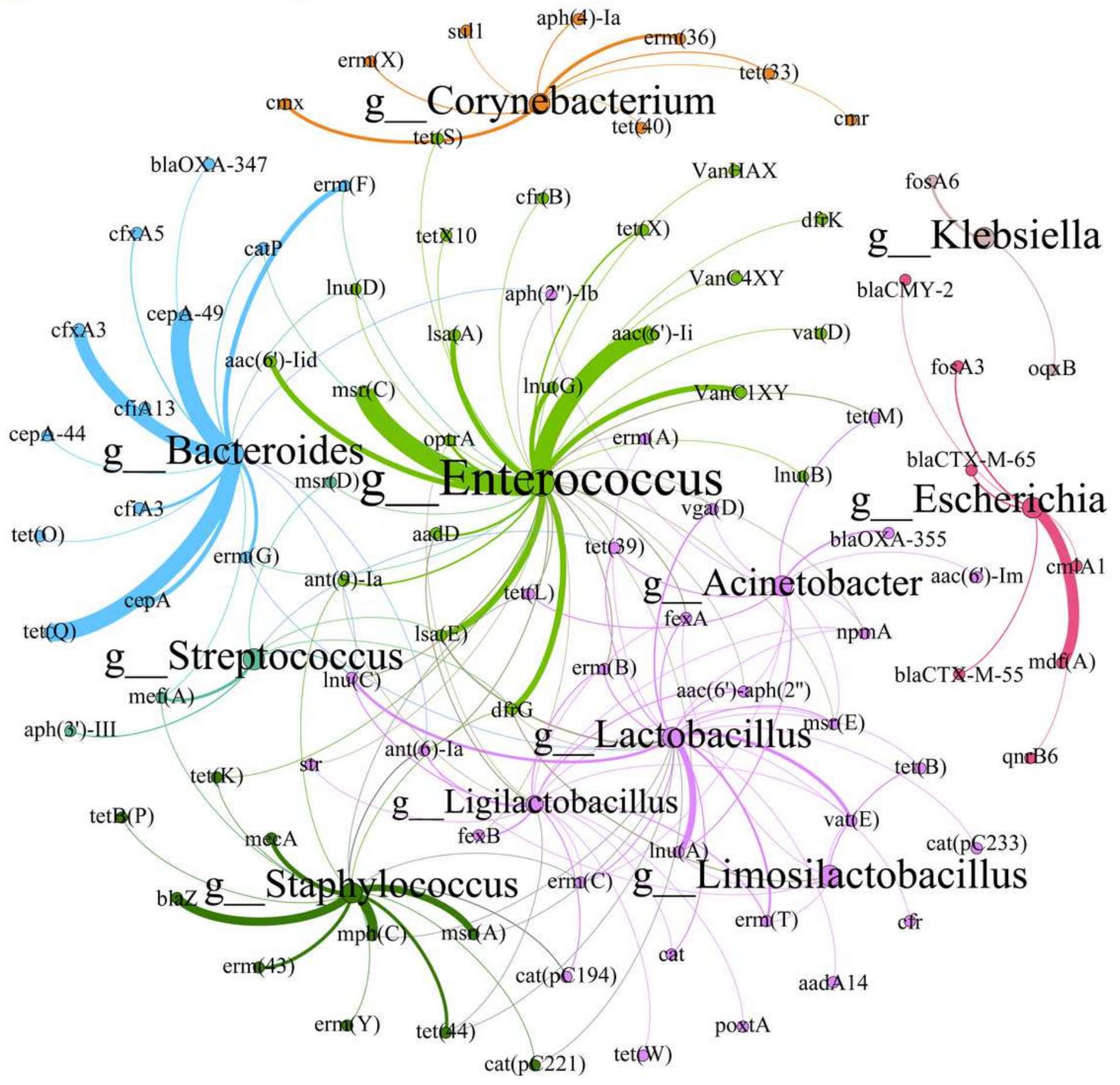

Figure 7

The network analysis revealing the co-occurrence patterns between major MAGs(in genus level) and ARG subtypes. The nodes were colored according to the modularity class. Modularity index 0.68 . The size of each node was proportional to the number of connections, that is, the average weighted.

\section{Supplementary Files}


This is a list of supplementary files associated with this preprint. Click to download.

- SupplementaryfileTableS.xlsx

- SupplementaryFig1.pdf

- SupplementaryFig2.pdf

- SupplementaryFig3.pdf 